\title{
Modern work patterns of "classic" versus millennial family doctors and their effect on workforce planning for community-based primary care: a cross-sectional survey
}

\author{
Lindsay Hedden ${ }^{1,2^{*}}$ (D), Setareh Banihosseini ${ }^{3}$, Nardia Strydom ${ }^{3,4,5}$ and Rita McCracken ${ }^{3,4}$
}

\begin{abstract}
Background: There are ongoing accessibility challenges in primary care in British Columbia, Canada, with 17\% of the population not having a regular source of care. Anecdotal evidence suggests that physicians are moving away from a community-based comprehensive practice model, which could contribute to shortages. Thus, we aimed to identify and describe how family physicians are currently organizing their primary care practices in a large health region in British Columbia and to examine differences between newer graduates and more established physicians.

Methods: Data for this cross-sectional study were drawn from an annual physician privileging survey. $N=$ 1017 physicians were invited to participate. We categorized practice style into five distinct groupings and compared features across respondent groups, including personal and practice location characteristics, hospital and teaching work, payment and appointment characteristics, and scope of practice. We discuss the implications of styles of practice and associated characteristics on health workforce policy and planning.

Results: We received responses from 525 (51.6\%) physicians. Of these, 355 (67.6\%) reported doing at least some community-based primary care. However, only 112 (21.3\%) provided this care full time. Most respondents supplemented community-based work with part-time hours in focused practice, hospitals, or inpatient facilities. We found diversity in the scope and style of practice across practice models. Compared to established physicians, new graduates (in practice less than 10 years) work more weekly hours (more patient care, and paperwork in particular). However, we found no difference between new and established physicians in the odds of providing any or full-time community-based primary care.

Conclusions: Despite a lack of formalized structural reform in British Columbia's primary care system, most physicians are finding alternative ways to model their practice and shifting away from work at single-location, community-based clinics. This shift challenges assumptions that are relied on for workplace planning that is intended to ensure adequate access to longitudinal, community-based family medicine.
\end{abstract}

\footnotetext{
* Correspondence: Lindsay_hedden@sfu.ca

${ }^{1}$ Faculty of Health Sciences, Simon Fraser University, Blusson Hall, Room

11300, 8888 University Drive, Burnaby, BC V5A 1S6, Canada

${ }^{2}$ British Columbia Academic Health Science Network, Vancouver, Canada

Full list of author information is available at the end of the article
}

C C The Author(s). 2020 Open Access This article is licensed under a Creative Commons Attribution 4.0 International License, which permits use, sharing, adaptation, distribution and reproduction in any medium or format, as long as you give appropriate credit to the original author(s) and the source, provide a link to the Creative Commons licence, and indicate if changes were made. The images or other third party material in this article are included in the article's Creative Commons licence, unless indicated otherwise in a credit line to the material. If material is not included in the article's Creative Commons licence and your intended use is not permitted by statutory regulation or exceeds the permitted use, you will need to obtain permission directly from the copyright holder. To view a copy of this licence, visit http://creativecommons.org/licenses/by/4.0/. The Creative Commons Public Domain Dedication waiver (http://creativecommons.org/publicdomain/zero/1.0/) applies to the data made available in this article, unless otherwise stated in a credit line to the data. 


\section{Background}

Approximately one in six British Columbians do not have a regular family doctor [1], matching the Canadian national average. News reports of patients looking for a family physician and being unable to find one are ubiquitous, and most physician practices are not currently accepting new patients [2-6]. The gap between demand and supply exists despite a consistent increase in numbers of family physicians per capita since 1986 [7, 8], and stable per-physician remuneration $[9,10]$. This suggests that either underlying changes in practice are reducing supply or demand is increasing.

Since federal Medicare legislation was passed in 1966, there have been no substantive structural changes to how primary care is delivered in British Columbia (BC), and it is assumed that the vast majority of physicians are practicing in the "classic" model. We define this as working in a community-based physician-owned and physician-operated practice, either alone or with a small group of physician colleagues, providing comprehensive full-scope care to a large panel of patients under a feefor-service (FFS) model [11]. Non-physician providers such as registered nurses, nurse practitioners, or other health professionals are not part of classic practices and have not been integrated into the primary care system more broadly.

There are signals, however, that the classic primary care model may no longer reflect how the majority of primary care physicians are practicing, and therefore that measures of supply within the system could be substantially inflated [12]. This could at least partly explain the conflicting observation of both increasing demand and increasing supply. Activity levels and patient panel sizes have declined substantially over time [10]. Fewer physicians are managing inpatient care for their own patients, or are otherwise providing services at non-officebased locations [13, 14], and comprehensiveness of care appears to be declining $[14,15]$.

Furthermore, there are options available for physicians who do not wish to run a solo or shared private community-based practice, such as hospitalist positions or locum tenens [16-18], and these roles are becoming more available. Physicians may choose to work in parttime "focused practice" type roles (e.g., sports medicine, palliative care, addiction treatment) [19].

There is also a small but growing body of evidence that suggests that new physicians are structuring their practices differently than more established physicians and that they may be more likely to choose some of these non-classic roles. For example, only $61 \%$ of family medicine residents in Western Canada stated that they intended to provide comprehensive care, and a full $28 \%$ report that they will focus their practice in specific clinical areas only [19]. Newer physicians provide a different basket of services than their more established counterparts $[15,20]$. They are also more likely to prefer nonFFS remuneration and to choose a model that does not involve clinic ownership and management [21, 22].

If physicians working in blended models, clinically focused practice, or full-time hospitalist or locum positions account for a substantial percentage of overall supply of primary care physicians, and particularly if these models are more common among newer-topractice physicians, there will be fewer physicians remaining to provide longitudinal, comprehensive, community-based primary care now and increasingly so in future. This could help to explain the co-occurring observations of increasing supply with no change in unmet need, but we are unaware of any studies that have examined this either in $\mathrm{BC}$ or elsewhere in Canada. The objectives of this cross-sectional survey analysis were to identify and describe current models of primary care practice in a Health Authority in $\mathrm{BC}$ and to examine whether and in what ways newer graduates model their practices differently than more established physicians.

\section{Methods}

\section{Setting and context}

Primary care in BC, Canada, is publicly funded and privately delivered. Primary care physicians, who deliver the vast majority of care, have traditionally worked in solo or small-group physician-owned and physician-operated practices. They are paid by the provincial health insurance plan on an FFS basis. Physicians pay overhead and staffing costs from gross FFS billings. There is a limited role for interdisciplinary teams or non-physician providers such as registered nurses and nurse practitioners. This is beginning to shift as the Ministry of Health implements some structural reforms (though these occurred after the data for this study were collected) [23-25].

$\mathrm{BC}$ is divided into five health regions. The largest region by population, Vancouver Coastal Health (VCH), provides services to 1.25 million British Columbians (25\% of the provincial population) across 12 municipalities and four regional districts. It operates a network of hospitals and acute care facilities, specialized community health centers, residential care facilities, and home support services.

Data for this cross-sectional study were drawn from information that is routinely collected as part of VCH's annual privileging process, which physicians are required to complete to confirm their eligibility to provide any services at a $\mathrm{VCH}$ facility. The research team designed a survey to address the annual privileging and workforce planning needs within $\mathrm{VCH}$ and to elicit physician perspectives on their models of practice. The survey was 
reviewed and approved by the Regional Medical Director of Primary Care for $\mathrm{VCH}$.

The survey captures information on how physicians are currently structuring their practices, and asks additional questions that support health authority-wide human resource planning such as planned retirements. The data were linked with publicly available demographic and training information from the College of Physicians and Surgeons of BC, including gender, year of graduation, and training location. While the privileging process is required, the survey component is optional.

Physicians who had clinical privileges with $\mathrm{VCH}$ in 2018 were invited (via email) to participate in the survey. Participation reminders were sent 1 and 5 weeks following the initial invitation. Responses were collected between January 30 and April 15, 2018. Six weeks after the initial invitation, physicians were also invited to have their data removed if they had reconsidered since first answering the survey. The survey was delivered online using REDCap. Researchers were presented with deidentified data for analysis.

\section{Survey content and variables}

We asked respondents to select between four options that describe their main model of primary care practice: all or some community-based primary care (CBPC), hospital or inpatient facility only, locum only, or nonclinical (provide no patient care). We grouped those respondents who indicated that they provide at least some CBPC based on the number of self-reported hours per week spent working in locations where CBPC is provided (solo, two to four, or five + physician clinics in the community that a patient can access without a referral). The three groups used were full-time CBPC $(>37.5 \mathrm{~h}$ per week), mostly CBPC plus some other work (20-37.5 $\mathrm{h}$ per week $\mathrm{CBPC}$ ), and mostly other work plus some CBPC $(<20 \mathrm{~h}$ per week CBPC). We also asked that they indicate whether their main model of practice was focused, general, or mixed (general but also with a specific clinical focus), and asked about the provision of specific services and care to special populations.

The survey also included a core set of demographic and work questions, including hours worked per "typical" week (broken into specific tasks areas such as patient care, paperwork, and business activities), number of practice locations, retirement intentions, and responsibilities for call coverage.

\section{Statistical analyses}

We excluded respondents who reported that they do not provide any patient care or who did not respond to core questions about model of practice. To determine the representativeness of our sample, we compared demographic (year of graduation and gender) and training (location) characteristics for survey respondents with the total population of primary care physicians practicing within $\mathrm{BC}$ area, using $\chi^{2}$ tests. These data are presented in Supplement 2. Data for this comparison were drawn from the College of Physicians and Surgeons of BC public listing.

For descriptive analysis, we used one-way ANOVA and $\chi^{2}$ tests to compare demographic and practice characteristics across core models of practice (some/all CBPC, hospital/facility only, or locum) and among physicians who provide at least some CBPC (less than half time, more than half time, or full time). We used the same test to compare demographic and practice characteristics between new graduates (physicians in practice less than 10 years) and more established physicians.

To assess the adjusted effect of demographic and practice characteristics on model of practice, we used a twostage modeling approach. We used a multivariable logistic model to assess which variables were associated with providing at least some CBPC. Then among the subsample of physicians who did report that they provide at least some CBPC, we constructed a second logistic model to assess the effect of the same set of demographic and practice characteristics on whether or not they provide CBPC care full time.

Finally, we conducted a series of $x^{2}$ tests looking for differences in provision of specific clinical services across full time, greater than half time, and less than half time spent providing CBPC. Services included maternity care, substance abuse treatments, non-office-based care, serving special populations, and the use of technology to support accessible patient care. All analyses were conducted in Stata IC/15.1.

\section{Results}

In total, 1017 physicians were initially invited to participate in the study. Of these, 584 physicians completed the survey (57.4\%; Fig. 1); however, 38 (6.5\%) of them subsequently requested that their data be withdrawn. An additional nine $(0.9 \%)$ responses were removed for physicians reporting that they do not provide any patient care, and six $(0.6 \%)$ were discarded because of incomplete responses to core practice model questions, leaving a final sample of $525(51.6 \%)$. Of the respondents in that final sample, 56\% (291) are women, $21.3 \%$ (112) trained outside of Canada, and $21.1 \%$ (111) provide at least some care in a rural practice location.

On average, respondents had been in practice for 22.5 years $(\mathrm{SD}=13.3)$, and $131(25.0 \%)$ intend to retire within the next 5 years. Respondents work an average of $44.1 \mathrm{~h}$ per week $(S D=15.2)$, and 30 of those hours $(S D=13.1)$ were dedicated to direct patient care.

Among respondents, 355 (67.6\%) reported doing at least some community-based primary care: 112 (31.6\%) 


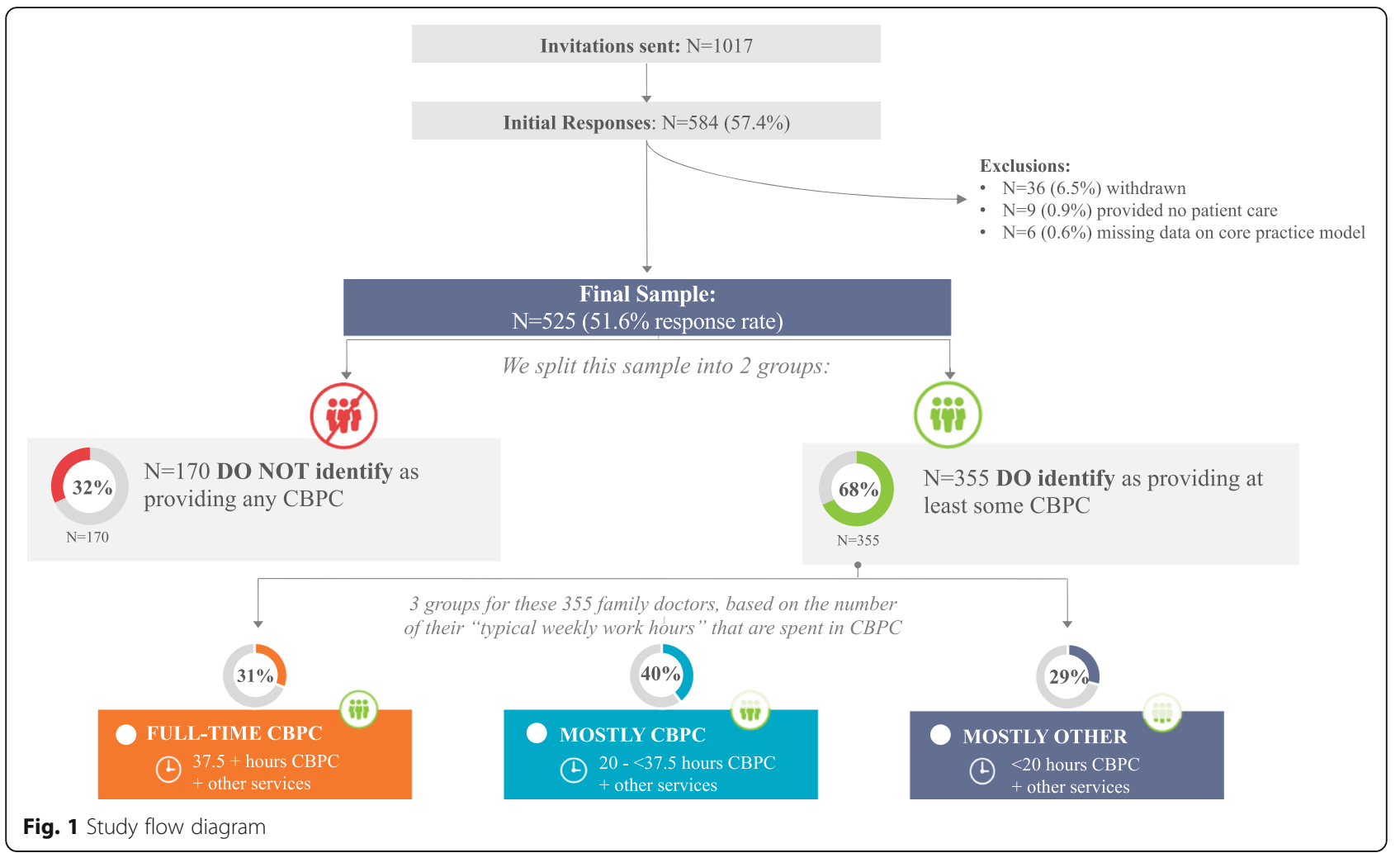

provide these services $37.5 \mathrm{~h}$ per week or more, 141 (39.7\%) between 20 and $37.5 \mathrm{~h}$ per week, and 102 (28.7\%) less than $20 \mathrm{~h}$ per week (Table 1). On average, physicians who reported that their model of practice includes at least some CBPC spent $27 \mathrm{~h}$ per week (SD = $16.0)$ on that work and $18.9 \mathrm{~h}$ per week $(\mathrm{SD}=15.7)$ on other work. Of respondents, 42 (11.8\%) provide no services in $\mathrm{CBPC}$ clinics, despite selecting this as their core model of practice. Among the remaining 170 who reported not providing CBPC, 97 (18.5\%) report working entirely in a hospital or inpatient facility and 73 (13.9\%) are locums.

At the bivariate level, we observed significant differences among practice models with respect to gender distribution, average years in practice, training location, rural practice location, work hours, and number of practice locations. We observed no difference in provision of call coverage or retirement planning (Table 1). Among the three groups of respondents who provided at least some CPBC, we observed significant bivariate differences in patient panel size; in focused, mixed, or general practice; and in working in at least one hospital/facility or emergency room. More physicians who provide CBPC for less than $20 \mathrm{~h}$ per week reported working in clinically focused practice compared with those who work between 20 and $37.5 \mathrm{~h}$ per week or more than $37.5 \mathrm{~h}$ per week (20.2\%, compared to $4.3 \%$ and $1.8 \%$ respectively). More physicians who work less than $20 \mathrm{~h}$ per week also had small patient panel sizes, but work at more locations than those who spend more time providing CBPC.

We found some differences in populations and services across the CBPC groups (Tables 2 and 3). More physicians providing CBPC full time reported providing prenatal and postpartum care than either part-time group. More physicians in this group also reported providing house calls, hospital visits, and residential care, and caring for elders with frailty. A significantly larger percentage of physicians who reported spending less than $20 \mathrm{~h}$ per week working in CBPC provide services for individuals with substance use disorders, including treating those disorders and prescribing methadone and buprenorphine/naloxone.

New graduates work more weekly hours (more patient care, and paperwork in particular) than established physicians. They also work in more locations (Table 4). We also found differences between new and established physicians in terms of model of practice, with more new graduates working in locum practice and fewer full-time or mostly in CPBC. New graduates are also more likely to work in mixed practice and less likely to work in focused or general practice models.

Our multivariable analyses found that physicians who trained internationally had significantly higher odds of reporting that some or all of their work is CBPC (some CBPC: OR 2.92, 95\% CI 1.70-5.01; full-time CPBC: OR 2.03, 95\% CI 1.20-3.43) than physicians who trained in 


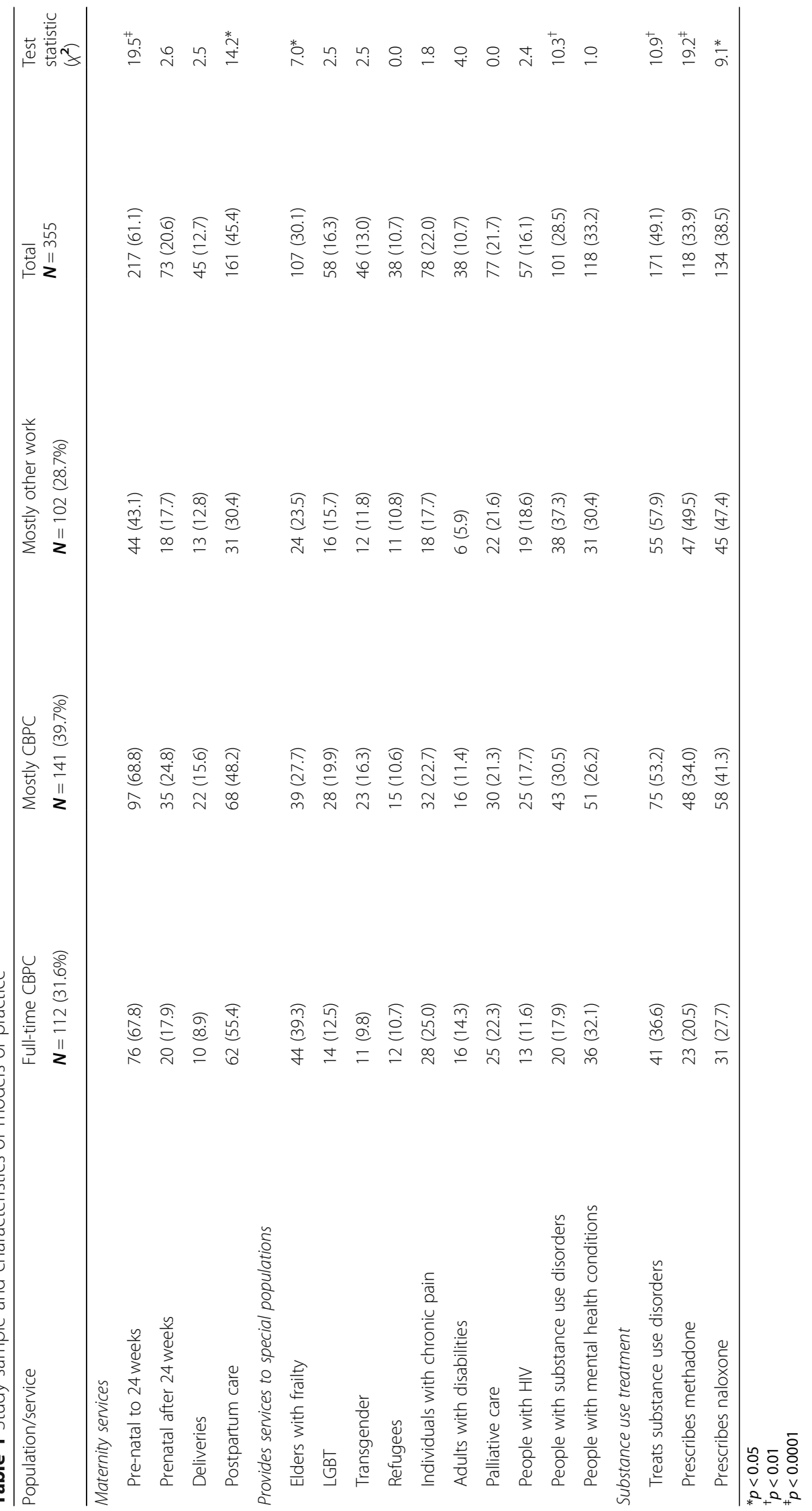




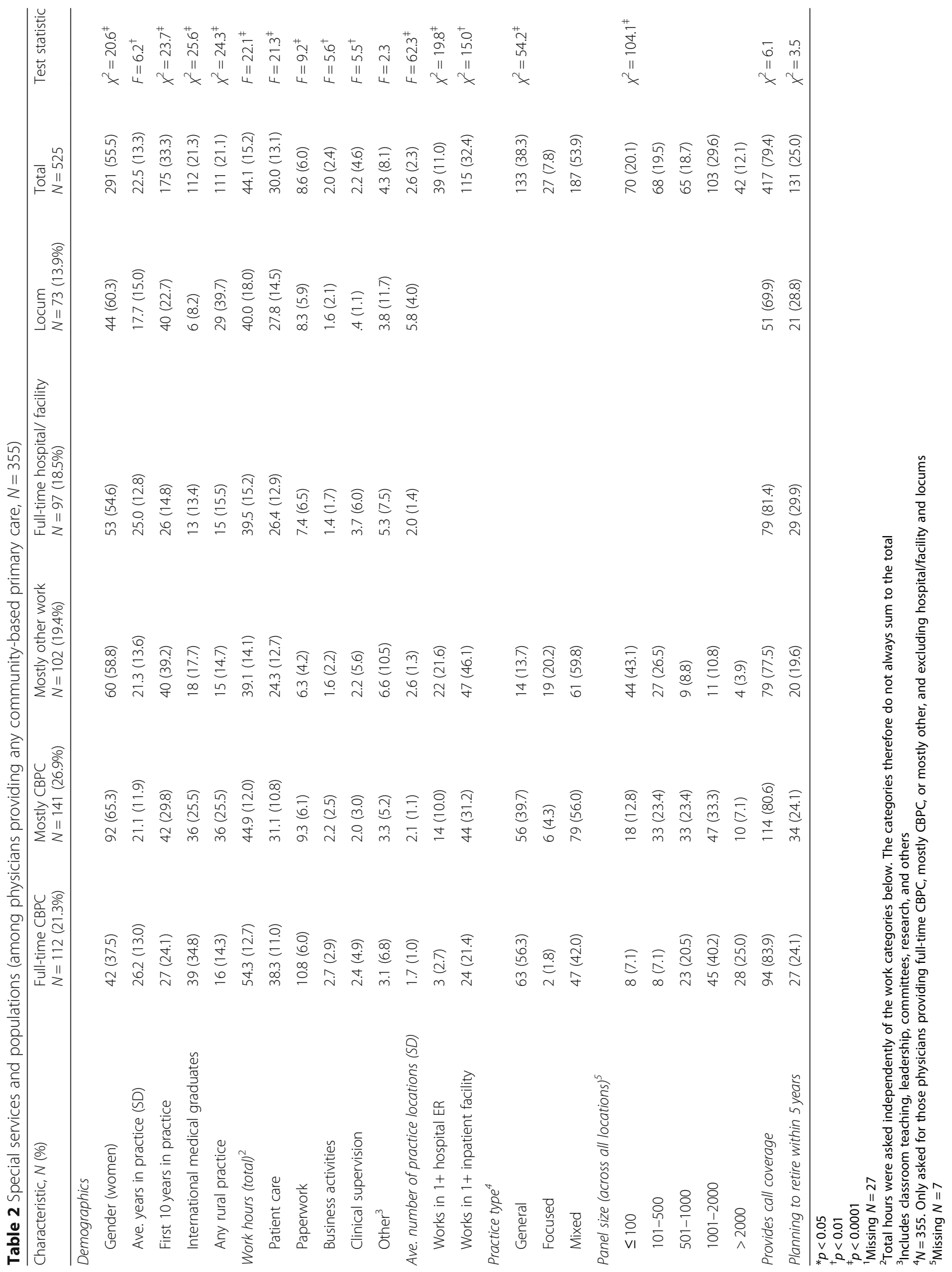


Table 3 Off-site care and technology for patient appointments (among physicians providing any community-based primary care, $N=355)$

\begin{tabular}{|c|c|c|c|c|c|}
\hline \multirow[t]{2}{*}{ Population/service } & \multirow{2}{*}{$\begin{array}{l}\text { Full-time CBPC } \\
\mathbf{N}=112(31.6 \%)\end{array}$} & \multirow{2}{*}{$\begin{array}{l}\text { Mostly CBPC } \\
\boldsymbol{N}=141(39.7 \%)\end{array}$} & \multirow{2}{*}{$\begin{array}{l}\text { Mostly other work } \\
\mathbf{N}=102(28.7 \%)\end{array}$} & \multirow{2}{*}{$\begin{array}{l}\text { Total } \\
\boldsymbol{N}=355\end{array}$} & \multirow{2}{*}{$\begin{array}{l}\text { Test } \\
\text { statistic }\end{array}$} \\
\hline & & & & & \\
\hline \multicolumn{6}{|l|}{ Offers any off-site visits } \\
\hline House calls & $80(71.4)$ & $96(68.1)$ & $47(46.1)$ & $223(62.8)$ & $17.5^{\ddagger}$ \\
\hline Hospital visits & $79(70.5)$ & $89(63.1)$ & $48(47.1)$ & $216(60.9)$ & $12.9^{\dagger}$ \\
\hline Residential care visits & $56(50.0)$ & $69(48.9)$ & $24(23.5)$ & $149(42)$ & $20.0^{\ddagger}$ \\
\hline Outreach services & $12(10.7)$ & $25(17.7)$ & $14(13.7)$ & $51(14.4)$ & 2.5 \\
\hline Care in schools & $4(3.6)$ & $3(2.1)$ & $4(3.9)$ & $11(3.1)$ & 0.8 \\
\hline Correctional facilities & $1(0.9)$ & $1(0.7)$ & $2(2.0)$ & $4(1.1)$ & 0.9 \\
\hline Other & $2(1.8)$ & $4(2.8)$ & $5(4.9)$ & $11(3.1)$ & 1.8 \\
\hline \multicolumn{6}{|l|}{ Technology for patient care } \\
\hline Email & $49(43.8)$ & $47(33.3)$ & $28(27.5)$ & $124(34.9)$ & $6.5^{*}$ \\
\hline Phone & $107(95.5)$ & $134(95.0)$ & $84(82.4)$ & $325(91.6)$ & $15.7^{\ddagger}$ \\
\hline Text message & $28(25.0)$ & $28(19.9)$ & $14(13.7)$ & $70(19.7)$ & 4.3 \\
\hline Skype/videoconference & $28(25.0)$ & $10(7.0)$ & $6(6.4)$ & 70 (19.7) & 0.9 \\
\hline
\end{tabular}

Canada (Table 5). Women had significantly lower odds of providing full-time CBPC (OR 0.37 , 95\% CI $0.23-$ 0.59 ). There was no significant difference in the odds of providing any or full-time CBPC between new or established family physicians once we adjusted for the effects of gender, training location, and practice location.

\section{Discussion}

We found that while two thirds of our respondents reported providing at least some $\mathrm{CBPC}$, only $21 \%$ of the sample provide that care full time. This substantial move away from the classic general practitioner model suggests that capacity for longitudinal CBPC is much more limited than headcount metrics or billings-based full-time equivalents would suggest. At the same time, only 5\% of our sample are working less than $20 \mathrm{~h}$ per week, suggesting that those who are spending less than $37.5 \mathrm{~h}$ per week doing CBPC are working full-time hours but spending time in non-CBPC locations such as focused practices, inpatient facilities, or emergency rooms (ERs). Furthermore, new graduates work on average $3.5 \mathrm{~h}$ per week more than established physicians, in contrast to the commonly heard narrative of millennial family doctors working part time and being a cause for erosion our primary care capacity [26].

This study is one of the few available descriptions of modern work patterns by family physicians in Canada. Existing literature relies heavily on billings data, which has not allowed for description of practice across multiple locations, fails to distinguish between community- based and other venues for delivering primary care, and cannot be used to calculate hours spent working. Even full-time equivalent calculations based on dollars billed, such as those produced by CIHI [27], do not account for whether the work a physician is doing is CBPC, hospitalist work, locum work, or something else. It is also one of the few studies that examine differences in work models and patterns between new graduates and established physicians. While it supports the existing finding that substantial numbers of new graduates do not (or do not intend to) provide comprehensive primary care [28, 29], it extends that work by providing direct comparison with more established physicians.

Our results also suggest that primary care physicians are finding alternative ways to model their practices, shifting away from single-location community-based clinics, despite the lack of formalized structural reform in BC's primary care system. Assessments of whether these new work patterns are aligned with patient need and what is driving physicians to move away from CBPC are beyond the scope of this paper, but both are critical areas of study that should be undertaken in the future. At a minimum, however, the fact that there is a substantial ongoing population need for increased access to longitudinal CBPC suggests that greater attention needs to be paid to what jobs are being offered to family physicians and how they compare to the models of practice physicians are creating for themselves.

Other ways to address the unmet need for primary care may involve a move to an interdisciplinary teambased model of care and formally incorporating 
Table 4 Comparison between new and established physicians

\begin{tabular}{|c|c|c|c|c|}
\hline Characteristic, N (column \%) & $\begin{array}{l}\text { New graduate } \\
N=175(33.3 \%)\end{array}$ & $\begin{array}{l}\text { Established } \\
N=350(66.7 \%)\end{array}$ & $\begin{array}{l}\text { Total } \\
N=525\end{array}$ & Test statistic \\
\hline \multicolumn{5}{|l|}{ Demographics } \\
\hline Gender (women) & $108(61.7)$ & $183(52.4)$ & $291(55.5)$ & $x^{2}=4.06^{*}$ \\
\hline International medical graduates & $27(15.4)$ & $85(24.3)$ & $112(21.3)$ & $x^{2}=5.45^{*}$ \\
\hline Any rural practice & $129(73.7)$ & $285(81.4)$ & $414(78.9)$ & $x^{2}=4.16^{*}$ \\
\hline Work hours (total) ${ }^{7}$ & $46.2(15.0)$ & $43.0(15.2)$ & $44.1(15.2)$ & $F=5.11^{*}$ \\
\hline Patient care & $31.2(12.8)$ & $29.4(13.2)$ & $30.0(13.1)$ & $F=2.24$ \\
\hline Paperwork & $8.9(5.5)$ & $8.4(6.3)$ & $8.6(6.0)$ & $F=0.91$ \\
\hline Business activities & $1.9(2.3)$ & $2.0(2.4)$ & $2.0(2.4)$ & $F=0.03$ \\
\hline Clinical supervision & $2.3(4.5)$ & $2.2(4.7)$ & $2.2(4.6)$ & $F=0.00$ \\
\hline Other ${ }^{2}$ & $3.9(8.9)$ & $4.6(7.7)$ & $4.3(8.1)$ & $F=0.72$ \\
\hline Practice model & & & & $x^{2}=23.67^{\ddagger}$ \\
\hline Full-time CBPC & $27(15.4)$ & $85(24.3)$ & $112(21.3)$ & \\
\hline Mostly CBPC & $42(24.0)$ & $99(28.3)$ & $141(26.9)$ & \\
\hline Mostly other & $40(22.9)$ & $62(17.1)$ & $102(19.4)$ & \\
\hline Hospital/facility & $26(14.9)$ & $71(20.3)$ & $97(18.5)$ & \\
\hline Locum & $40(22.9)$ & $33(9.4)$ & $73(13.9)$ & \\
\hline Ave. number of practice locations (SD) & $2.6(1.2)$ & $1.9(1.1)$ & $2.1(1.2)$ & $F=46.6^{\ddagger}$ \\
\hline Works in $1+$ hospital ER & $16(14.7)$ & $23(9.35)$ & $39(11.0)$ & $x^{2}=2.19$ \\
\hline Works in $1+$ inpatient facility & $50(45.9)$ & $65(26.4)$ & $115(32.4)$ & $x^{2}=13.05^{\ddagger}$ \\
\hline Works in $1+$ walk-in clinic & $31(11.8)$ & $29(11.8)$ & $60(16.9)$ & $x^{2}=9.27^{\dagger}$ \\
\hline Practice type $^{3}$ & & & & $x^{2}=5.18$ \\
\hline General & $65(61.3)$ & $122(50.6)$ & $187(53.9)$ & \\
\hline Focused & $4(3.8)$ & $23(9.5)$ & $27(7.8)$ & \\
\hline Mixed & $37(34.9)$ & $96(39.8)$ & $133(38.3)$ & \\
\hline Provides call coverage & $147(84.0)$ & $270(77.1)$ & $417(79.4)$ & $x^{2}=3.36$ \\
\hline
\end{tabular}

registered nurses, nurse practitioners, and other nonphysician providers into the primary care system. The BC Ministry of Health has recently started to implement a substantial suite of reforms that includes a core focus on interdisciplinary teams [23-25], organized into $\mathrm{Pa}$ tient Medical Homes [30]. The Patient Medical Homes will still be physician-owned and physician-operated, and FFS remuneration is expected to be the dominant form of remuneration. While in theory this could reduce the number of physicians required to meet population health needs, the extent of the uptake of these voluntary reforms among new graduates and established physicians

Table 5 Multivariate modeling results - predictors of models of primary care practice

\begin{tabular}{|c|c|c|}
\hline \multirow[t]{2}{*}{ Characteristic } & Model 1: Any CBPC & Model 2: Full-time CBPC* \\
\hline & Odds ratio $(95 \% \mathrm{Cl})$ & Odds ratio $(95 \% \mathrm{Cl})$ \\
\hline New graduate (< 10 years since graduation) & $0.79(0.53,1.17)$ & $0.72(0.42,1.22)$ \\
\hline Gender (women) & $0.94(0.64,1.37)$ & $0.37(0.23,0.59)$ \\
\hline Training (international) & $2.92(1.70,5.01)$ & $2.03(1.20,3.43)$ \\
\hline Practice location (rural) & $0.61(0.39,0.96)$ & $0.52(0.27,1.00)$ \\
\hline
\end{tabular}

*Locums, hospital/facility-based physicians excluded 
remains to be seen, as will the extent to which the reforms can successfully reduce the level of unmet need.

\section{Limitations}

This study used a cross-sectional survey-based analysis and has the standard set of limitations associated with that approach. Our respondents differ from the broader population of primary care physicians within $\mathrm{VCH}$ and BC. For example, women are over-represented in our sample (55.4\%) compared to all primary care physicians practicing within $\mathrm{VCH}$ (48.4\%) and across BC more broadly (42.0\%; Supplement 1). Our sample also had a smaller percentage of physicians who trained outside of Canada (21.8\%) compared to the larger BC physician population $(32.6 \%)$, but similar to the percentage for $\mathrm{VCH}(25.7 \%)$.

Given that women were over-represented in our sample, and the relationship we observed between gender and the odds of doing any or full-time CBPC, it is possible we are underestimating the proportion of physicians working in either of these models at least somewhat. Furthermore, because this survey was collected as part of annual privileging, it is likely we are overestimating the proportion of family physicians who work solely or in part in a hospital or facility. Hours spent providing $\mathrm{CPBC}$ are likely to be underestimated in a rural population, where primary clinics may be nested within or closely affiliated with a hospital. However, because $\mathrm{VCH}$ is a primarily urban health authority, we expect any effect on our results to be minimal.

\section{Conclusions}

This cross-sectional survey supports the growing body of evidence on access and capacity challenges in community-based primary care in British Columbia. Models of practice are moving away from full-time care in the community and toward models that blend community-based care with work in other practice locations. Approaches to workforce planning in the context of primary care need to account for shifting models of practice in their estimates of current and future primary care capacity. Furthermore, ongoing reforms to primary care, including the introduction of team-based Patient Medical Homes and Primary Care Networks, should be made with the intention of making community-based practice an attractive and viable option relative to hospitalist, locum, and blended practice models.

\section{Supplementary information}

Supplementary information accompanies this paper at https://doi.org/10. 1186/s12960-020-00508-5.

Additional file 1. Representativeness of study sample. Table S1.1: Comparison between study sample and all primary care physicians practicing within Vancouver Coastal Health. Table S1.2: Comparison between study sample and all primary care physicians practicing within British Columbia.

\section{Abbreviations}

ANOVA: Analysis of variance; BC: British Columbia; CBPC: Community-based primary care; ER: Emergency room; FFS: Fee-for-service; VCH: Vancouver Coastal Health

\section{Acknowledgements}

The authors wish to gratefully acknowledge the contributions of Kassra Hassani, Sara Zeinoddini, Ali Mokhtari Amirmajdi, Xi Kang, Parisa Khoshpouri, and Javad Ramezani on the Models and Access Atlas of Primary Care (MAAP) - BC Study.

\section{Authors' contributions}

LH developed the online survey tool, designed the analytic strategy, cleaned and analyzed all data, drafted the manuscript, and incorporated coauthor feedback. RM conceptualized the study, drafted the survey questions, and provided feedback on data analyses and manuscript development at all stages. SB assisted with conceptualization of the study and contributed to drafting the manuscript. NS assisted with conceptualizing the study and contributed to drafting the manuscript and interpretation of results. All authors read and approved the final manuscript.

\section{Funding}

This study was funded by Vancouver Coastal Health Authority. They assisted with the development of the survey tool but had no involvement in data collection, analyses, or the drafting of this manuscript.

\section{Availability of data and materials}

The data that support the findings of this study are available from Providence Health Care, but restrictions apply to availability. Data were used under license for the current study and so are not publicly available. Data are however available from the authors upon reasonable request and with permission of Providence Health Care.

\section{Ethics approval and consent to participate}

Ethics approval was waived by the University of British Columbia Providence Health Care Research Ethics Board as the survey data were collected for a primary purpose other than research. Participants were provided with introductory material explaining that their participation in the survey component of the credentialing process was voluntary and that data would be aggregated and anonymized. They were also provided the opportunity to withdraw consent and have their responses removed from the dataset should they change their mind after filling out the survey.

Consent for publication

Not applicable

\section{Competing interests}

The authors declare that they have no competing interests.

\section{Author details}

${ }^{1}$ Faculty of Health Sciences, Simon Fraser University, Blusson Hall, Room 11300, 8888 University Drive, Burnaby, BC V5A 1S6, Canada. ${ }^{2}$ British Columbia Academic Health Science Network, Vancouver, Canada. ${ }^{3}$ Department of Family Medicine, Providence Health Care, Vancouver, Canada. ${ }^{4}$ Department of Family Practice, Faculty of Medicine, University of British Columbia, Vancouver, Canada. ${ }^{5}$ Department of Family and Community Medicine, Vancouver Coastal Health, Vancouver, Canada.

Received: 7 November 2019 Accepted: 27 August 2020

Published online: 21 September 2020

\section{References}

1. Canadian Institute for Health Information. Primary health care in Canada: a chartbook of selected indicator results, 2016. 2016. 
2. Strandberg D. Doctor shortage blamed for long waits at Tri-City clinics. TriCity News. [cited 2020 May 1]; Available from: https://www.tricitynews.com/ news/doctor-shortage-blamed-for-long-waits-at-tri-city-clinics-1.24034929.

3. Michelle Morton. No end in sight to B.C.'s doctor shortage as major online resource disappears. Global News. [cited 2020 May 1]; Available from: https://globalnews.ca/news/4064761/bc-doctor-shortage/.

4. Lau L. Why is it so hard to find a family doctor in Vancouver? Vancouver Magazine. [cited 2020 May 1]; Available from: https://www.vanmag.com/ Why-Is-it-so-Hard-to-Find-a-Doctor-in-Vancouver.

5. Nick Murray. Doctor shortage frustrating south Island residents. Vancouver Island Free Daily. 2019 Aug 13 [cited 2020 May 1]; Available from: https:// www.vancouverislandfreedaily.com/news/doctor-shortage-frustrating-southisland-residents/.

6. Harnett LK Cindy E. Why can't you find a doctor? Times Colonist. [cited 2020 May 1]; Available from: https://www.timescolonist.com/islander/whycan-t-you-find-a-doctor-1.23863824.

7. Canadian Institute for Health Information. Scott's Medical Database - Data Tables. Ottawa: $\mathrm{ClHI} ; 2017$.

8. Canadian Institute for Health Information. Physicians in Canada. Ottawa; 2019.

9. Canadian Institute for Health Information. Supply, Distribution and Migration of Physicians in Canada, 2017. Ottawa; 2018.

10. Canadian Institute for Health Information. More doctors, but average payments to physicians virtually unchanged | CIHI. 2016 [cited 2016 Aug 28]. Available from: https://www.cihi.ca/en/spending-and-health-workforce/ health-workforce/more-doctors-but-average-payments-to-physicians.

11. Hutchison B, Levesque J-F, Strumpf E, Coyle N. Primary health care in Canada: systems in motion. Milbank Q. 2011;89(2):256-88.

12. Hedden L, Barer ML, Mc Grail K, Law M, Bourgeault ILIL. In British Columbia, the supply of primary care physicians grew, but their rate of clinical activity declined. Health Aff (Millwood). 2017;11(11):1904-11.

13. Hedden L, Lavergne MR, Mc Grail KM, Law MR, Bourgeault IL, Mc Cracken R, et al. Trends in providing out-of-office, urgent after-hours, and on-call care in British Columbia. Ann Fam Med. 2019;17(2):116-24.

14. Chan BTB. The declining comprehensiveness of primary care. CMAJ. 2002; 166(4):429-34.

15. Lavergne MR, Peterson S, Mc Kendry R, Sivananthan S, Mc Grail K. Fullservice family practice in British Columbia: policy interventions and trends in practice, 1991-2010. Healthc Policy. 2014;9(4):32-47.

16. Wachter RM, Bell D. Renaissance of hospital generalists. BMJ. 2012

17. White HL, Stukel TA, Wodchis WP, Glazier RH. Defining hospitalist physicians using clinical practice data: a systems-level pilot study of Ontario physicians. 2013;7(3)::74-84.

18. Soong C, Fan E, Howell EE, Maloney RJ, Pronovost PJ, Wilton D, et al. Characteristics of hospitalists and hospitalist programs in the united states and canada. J Clin Outcomes Manag. 2009.

19. Schultz SE, Glazier RH. Identification of physicians providing comprehensive primary care in Ontario: a retrospective analysis using linked administrative data. CMAJ Open. 2017;5(4):E856-63.

20. Mc Grail K, Lavergne R, Lewis S, Peterson S, Barer M, Garrison S. Classifying physician practice style: a new approach using administrative data in British Columbia. Med Care. 2015;53(3):276-82.

21. Hedden L, Strydom N, Mc Cracken R. Models of primary care practice and priorities for reform: a survey based analysis. In Halifax; 2019.

22. Brcic V, MJ MG, Kaczorowski J, Dharamsi S, Verma S. Practice and payment preferences of newly practising family physicians in British Columbia. Can Fam Physician. 2012;58(5):e275-81.

23. British Columbia Ministry of Health. General Policy Direction - Primary Care Networks: British Columbia Ministry of Health; 2017.

24. British Columbia Ministry of Health. Supportive Policy Direction Interdisciplinary Team Based Care: British Columbia Ministry of Health; 2017.

25. British Columbia Ministry of Health. Supportive Policy Direction - Patient Medical Homes: British Columbia Ministry of Health; 2017.

26. McElroy R. Erosion of comprehensive care and professionalism. Can Fam Physician. 2018;64(12):875-7.

27. Canadian Institute for Health Information. Approaches for calculating average clinical payments per physician using detailed alternative payment data spending and health workforce. 2015.

28. Lavergne MR, Scott I, Mitra G, Snadden D, Blackie D, Goldsmith LJ, et al. Regional differences in where and how family medicine residents intend to practise: a cross-sectional survey analysis. CMAJ Open. 2019;7(1):E124-30.
29. Lavergne MR, Gonzalez A, Ahuja MA, Hedden L, McCracken R. The relationship between gender, parenthood and practice intentions among family medicine residents: cross-sectional analysis of national Canadian survey data. Hum Resour Health. 2019;17(1):67.

30. Hefford B. The patient medical home: working together to create an integrated system of care. Br Columbia Med J. 2017;59(1):15-7.

\section{Publisher's Note}

Springer Nature remains neutral with regard to jurisdictional claims in published maps and institutional affiliations.
Ready to submit your research? Choose BMC and benefit from:

- fast, convenient online submission

- thorough peer review by experienced researchers in your field

- rapid publication on acceptance

- support for research data, including large and complex data types

- gold Open Access which fosters wider collaboration and increased citations

- maximum visibility for your research: over $100 \mathrm{M}$ website views per year

At BMC, research is always in progress.

Learn more biomedcentral.com/submissions 